\title{
Quark and Lepton Evolution Invariants in the Standard Model
}

\author{
P.F. Harrison* Department of Physics, University of Warwick, Coventry CV4 7AL, UK \\ E-mail: p.f.harrisonewarwick.ac.uk \\ R. Krishnan \\ Department of Physics, University of Warwick, Coventry CV4 7AL, UK \\ E-mail: K.Rama@warwick.ac.uk
}

\section{W.G. Scott}

Rutherford Appleton Laboratory, Chilton, Didcot, Oxon OX11 OQX, UK

E-mail: william.scottestfc.ac.uk

\begin{abstract}
We construct a set of exact Standard Model renormalisation group evolution invariants which link quark masses and mixing parameters in a completely new way. We examine their phenomenological implications and infer a simple combination of Yukawa coupling matrices which appears to play a unique role in the Standard Model. This suggests a possible new insight into the observed spectrum of quark masses. Similar evolution invariants are obtained for the leptons in the case of Dirac neutrinos.
\end{abstract}

35th International Conference of High Energy Physics

July 22-28, 2010

Paris, France

\footnotetext{
*Speaker.
} 


\section{Introduction}

There has recently been interest [1]-[4] in Renormalisation Group Invariants (RGIs), observables which do not evolve with energy. They may be useful since eg. their experimentallydetermined values and uncertainties, determined at one scale, are valid at all scales. They can also be useful, as we shall see, in identifying viable phenomenological relationships between observables, since once established among invariants, such relationships will be independent of energy. However, until now, only approximate invariants have been derived, using various simplifying assumptions. Moreover, much of the earlier emphasis has been on Beyond Standard Model examples, and surprisingly little work has been done along this line within the Standard Model (SM) itself. Here, we present our recently-derived [5] exact evolution invariants in the SM (at one-loop order), involving only the Yukawa couplings.

In the Standard Model (SM), quark and lepton masses and mixings originate in their Yukawa couplings to the Higgs. Considering quarks and leptons of a given charge, each possible pair can couple to the Higgs, each with arbitrary complex coupling. With 3 families of fermions, this gives arbitrary complex $3 \times 3$ matrices of Yukawa couplings, one for each charge of fermion, referred to here as $U, D, L$ and $N$ for charge $2 / 3,-1 / 3,-1$ and 0 respectively. Their eigenvalues are proportional to the (Dirac) masses of the fermions, while the quark and lepton mixing matrices are formed from the matrices which diagonalise them. We will find it convenient to work with the Hermitian squares of these Yukawa matrices:

$$
\mathscr{U}=U^{\dagger} U ; \quad \mathscr{D}=D^{\dagger} D
$$

The Yukawa couplings evolve with energy scale according to 1 st order differential renormalisation group equations (RGEs). The RGEs are usually written as matrix equations and at one-loop order are given [6] as:

$$
U^{-1} \frac{d U}{d t}=\gamma_{u}+\frac{3}{2}(\mathscr{U}-\mathscr{D}) ; \quad D^{-1} \frac{d D}{d t}=\gamma_{d}+\frac{3}{2}(\mathscr{D}-\mathscr{U})
$$

where

$$
\gamma_{u}=T-\left(\frac{17}{12} g_{1}^{2}+\frac{9}{4} g_{2}^{2}+8 g_{3}^{2},\right) ; \quad \gamma_{d}=T-\left(\frac{5}{12} g_{1}^{2}+\frac{9}{4} g_{2}^{2}+8 g_{3}^{2}\right),
$$

with:

$$
T=\operatorname{Tr}(3 \mathscr{U}+3 \mathscr{D}+\mathscr{N}+\mathscr{L})
$$

(the $g_{i}$ being the gauge couplings). These equations control how the quark masses and mixing angles evolve. Analogous equations are obtained for leptons in the Dirac neutrino case (with slightly different functions $\gamma_{\ell}$ and $\gamma_{v}$ ). The RGEs for the Hermitian squares, $\mathscr{U}$ and $\mathscr{D}$, are readily derived [5] from those above. The RGEs for the Yukawas are coupled and non-linear. Given measured values of the observables at e.g. the weak scale, the RGEs can be solved for observables at high (eg. GUT) scales. However, experimental uncertainties in poorly-known observables feed into the solutions for almost all other observables, rendering their high-energy values poorly-determined, and hampering attempts to establish patterns and relationships between parameters at high scales. 


\section{Pure Renormalisation Group Evolution}

We first consider the RG evolution of some typical SM flavour observables, the charge $\frac{2}{3}$ quark masses, proportional to the (square roots of the) eigenvalues $\lambda_{u i}$ of $\mathscr{U}$. The latter are given by the eigenvalue equation:

$$
\lambda_{u}^{3}-T_{\mathscr{U}} \cdot \lambda_{u}^{2}+P_{\mathscr{U}} \cdot \lambda_{u}-D_{\mathscr{U}}=0
$$

where the coefficients are:

$$
T_{\mathscr{U}}=\operatorname{Tr}(\mathscr{U}) ; \quad P_{\mathscr{U}}=\frac{1}{2}\left[\operatorname{Tr}^{2}(\mathscr{U})-\operatorname{Tr}\left(\mathscr{U}^{2}\right)\right] ; \quad D_{\mathscr{U}}=\operatorname{Det}(\mathscr{U}) .
$$

The RGEs of such flavour-symmetric functions of Yukawa matrices may be found exactly:

$$
\begin{aligned}
\frac{d T_{\mathscr{U}}}{d t} & =2 \gamma_{u} T_{\mathscr{U}}+3\left[T_{\mathscr{U}}^{2}-2 P_{\mathscr{U}}-\operatorname{Tr}(\mathscr{U} \mathscr{D})\right] \\
\frac{d P_{\mathscr{U}}}{d t} & =4 \gamma_{u} P_{\mathscr{U}}+3 P_{\mathscr{U}}\left(T_{\mathscr{U}}-T_{\mathscr{D}}\right)+3 D_{\mathscr{U}}\left[\operatorname{Tr}\left(\mathscr{U}^{-1} \mathscr{D}\right)-3\right] \\
\frac{d D_{\mathscr{U}}}{d t} & =3 D_{\mathscr{U}}\left(2 \gamma_{u}+T_{\mathscr{U}}-T_{\mathscr{D}}\right) .
\end{aligned}
$$

Similar evolution equations are valid for the analogous functions of $\mathscr{D}$ in the case of the charge $-\frac{1}{3}$ quarks.

The non-linearity and mutual coupling exhibited by the RGEs of $U$ and $D$, Eq. (1.2), is reflected in the above RGEs, confirming that the evolution of the individual eigenvalues depends in a complicated way on the eigenvalues of both $\mathscr{U}$ and $\mathscr{D}$, and indeed on the mixing observables themselves.

We now consider instead the eigenvalues, $\lambda_{i}$, of the product matrix $\mathscr{U} \mathscr{D}$. They are the solutions of its eigenvalue equation:

$$
\lambda^{3}-T_{\mathscr{U} \mathscr{D}} \cdot \lambda^{2}+P_{\mathscr{U} \mathscr{D}} \cdot \lambda-D_{\mathscr{U} \mathscr{D}}=0
$$

whose coefficients have similar definitions to those in Eq. (2.2) above. Starting with Eq. (1.2), it can be shown [5] that these coefficients have pure RG evolutions, by which we mean that the evolution is simply proportional to the variable itself (apart from the trivial coupling to others via the $\gamma$ functions, Eq. (1.3)):

$$
\begin{aligned}
& \frac{d T_{\mathscr{U} \mathscr{D}}}{d t}=2\left(\gamma_{u}+\gamma_{d}\right) T_{\mathscr{U} \mathscr{D}} \\
& \frac{d P_{\mathscr{U} \mathscr{D}}}{d t}=4\left(\gamma_{u}+\gamma_{d}\right) P_{\mathscr{U} \mathscr{D}} \\
& \frac{d D_{\mathscr{U} \mathscr{D}}}{d t}=6\left(\gamma_{u}+\gamma_{d}\right) D_{\mathscr{U} \mathscr{D} .}
\end{aligned}
$$

Moreover, their evolution rates in each case, Eqs. (2.7)-(2.9), are given simply by twice the dimensional order of the coefficient in question (c.f. Eq.(2.6)) times a common factor $\left(\gamma_{u}+\gamma_{d}\right)$. We conclude that the eigenvalues, $\lambda_{i}$, of the product matrix $\mathscr{U} \mathscr{D}$ have pure evolutions with coefficient $2\left(\gamma_{u}+\gamma_{d}\right)$. This is a new result. It appears then that the product matrix $\mathscr{U} \mathscr{D}$ plays a special role in the SM. This special role for the matrix $\mathscr{U} \mathscr{D}$ is traceable to the economical use in the SM of a single Higgs field to give masses to all the fermions. We have so far been unable to find any parallel result within the MSSM or two Higgs doublet model. 


\section{Exact Renormalisation Group Invariants in the SM}

We have seen that the three $\mathscr{U} \mathscr{D}$ coefficients, $T_{\mathscr{U} \mathscr{D}}, P_{\mathscr{U} \mathscr{D}}$ and $D_{\mathscr{U} \mathscr{D}}$ all have pure evolutions with rates proportional to their order. We may thus form from them two independent "dimensionless" combinations which are exact one-loop evolution invariants:

$$
\mathscr{I}_{T D}^{q} \equiv \frac{T_{\mathscr{U} \mathscr{D}}}{D_{\mathscr{U} \mathscr{D}}^{\frac{1}{3}}} ; \quad \mathscr{I}_{P D}^{q} \equiv \frac{P_{\mathscr{U} \mathscr{D}}}{D_{\mathscr{U} \mathscr{D}}^{\frac{2}{3}} ;} \quad \text { such that } \frac{d \mathscr{I}_{T D}^{q}}{d t}=\frac{d \mathscr{I}_{P D}^{q}}{d t}=0 .
$$

These invariants may be evaluated in terms of the conventional mass and mixing observables:

$$
\begin{aligned}
& \mathscr{I}_{T D}^{q}=\sum_{\alpha \neq \beta \neq \gamma, i \neq j \neq k}\left(\frac{m_{\alpha}^{2}}{m_{\beta} m_{\gamma}} \frac{m_{i}^{2}}{m_{j} m_{k}}\right)^{\frac{2}{3}}\left|V_{\alpha i}\right|^{2} \simeq\left(\frac{m_{t}}{m_{u}} \frac{m_{t}}{m_{c}} \frac{m_{b}}{m_{d}} \frac{m_{b}}{m_{s}}\right)^{\frac{2}{3}} \cos ^{2} \theta_{23} \sim 10^{8} ; \\
& \mathscr{I}_{P D}^{q}=\sum_{\alpha \neq \beta \neq \gamma, i \neq j \neq k}\left(\frac{m_{\beta} m_{\gamma}}{m_{\alpha}^{2}} \frac{m_{j} m_{k}}{m_{i}^{2}}\right)^{\frac{2}{3}}\left|V_{\alpha i}\right|^{2} \simeq\left(\frac{m_{t}}{m_{u}} \frac{m_{c}}{m_{u}} \frac{m_{b}}{m_{d}} \frac{m_{s}}{m_{d}}\right)^{\frac{2}{3}} \cos ^{2} \theta_{12} \sim 10^{8},
\end{aligned}
$$

where the last expression in each case is valid to leading order in small mass ratios. For the leptons, in the Dirac neutrino case, we find analogous evolution invariants, $\mathscr{I}_{T D}^{q} \rightarrow \mathscr{I}_{T D}^{\ell}$ and $\mathscr{I}_{P D}^{q} \rightarrow \mathscr{I}_{P D}^{\ell}$, with $\mathscr{U} \rightarrow \mathscr{N}$ and $\mathscr{D} \rightarrow \mathscr{L}, \gamma_{u} \rightarrow \gamma_{v}$ and $\gamma_{d} \rightarrow \gamma_{\ell}$.

In Fig. 1, we indicate the experimentally allowed ranges of values of $\mathscr{I}_{T D}^{q}$ and $\mathscr{I}_{P D}^{q}$, plotted against each other.

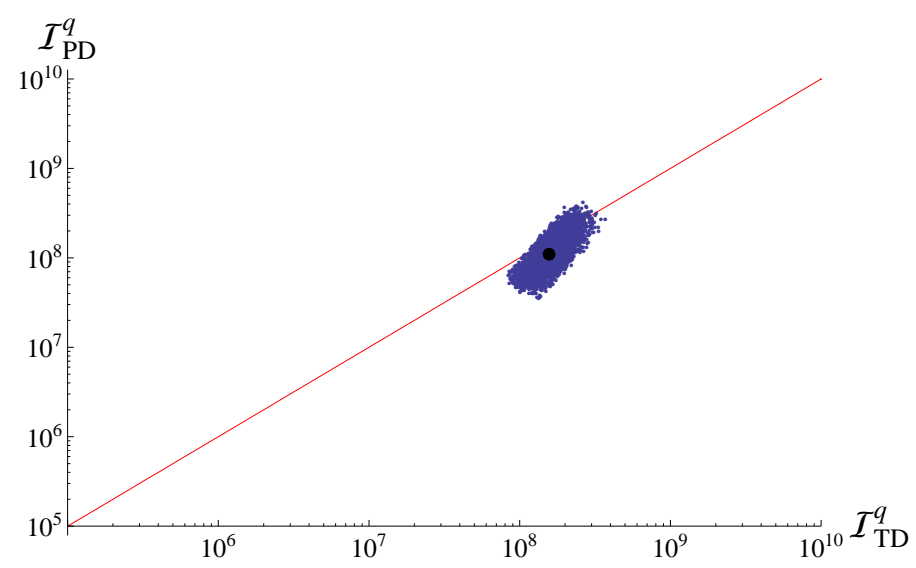

Figure 1: The black point shows the values of the RG invariants $\mathscr{I}_{T D}^{q}$ and $\mathscr{I}_{P D}^{q}$ evaluated using experimental values of quark masses and mixings, renormalised to the weak scale taken from [7]. The cluster of points indicates the range allowed by experimental and theoretical uncertainties. The straight line shows the hypothesis $\mathscr{I}_{T D}^{q}=\mathscr{I}_{P D}^{q}$ suggested by the data.

$\mathscr{I}_{T D}^{q}$ and $\mathscr{I}_{P D}^{q}$ are independent combinations of quark masses and mixings and could of course have taken any values in nature. Yet we have seen, Eqs. (3.2)-(3.3), that they are both $O\left(10^{8}\right)$ and that in fact:

$$
\frac{\mathscr{I}_{P D}^{q}}{\mathscr{I}_{T D}^{q}} \approx\left(\frac{m_{c}^{2}}{m_{t} m_{u}} \frac{m_{s}^{2}}{m_{b} m_{d}}\right)^{\frac{2}{3}} \frac{\cos ^{2} \theta_{23}}{\cos ^{2} \theta_{12}}=0.7_{-0.4}^{+1.1}
$$

a relation which is equally as valid at high scales as at the weak scale. It is of course a complete mystery why their measured values are so similar, this feature seeming to require an unnatural level 
of fine-tuning. The near equality, Eq. (3.4), prompts us to postulate that:

$$
\frac{\mathscr{I}_{P D}^{q}}{\mathscr{I}_{T D}^{q}} \equiv 1
$$

exactly. We have shown [5] that such an equality implies a geometric spectrum for the product matrix $\mathscr{U} \mathscr{D}$, ie.

$$
\mathscr{I}_{P D}^{q}=\mathscr{I}_{T D}^{q} \Rightarrow \frac{\lambda_{2}}{\lambda_{1}}=\frac{\lambda_{3}}{\lambda_{2}} .
$$

While for any given quark species, an approximate geometric spectrum of masses may be considered almost folklore, this is the first time that a quantitative postulate, Eq. (3.5), has been put forward, specifically in this case for the eigenvalues of the product matrix $\mathscr{U} \mathscr{D}$. Note that the eigenvalues of this matrix are not the same as the product of the eigenvalues of $\mathscr{U}$ and $\mathscr{D}$ separately, and thus our postulate cannot be tested using masses alone but necessarily involves mixing angles also, see Eq. (3.4).

Finally we also found two evolution invariants linking the Yukawa couplings of quarks and leptons together with the gauge couplings. These invariants are also new and, despite their somewhat contrived appearance, are reproduced here for completeness:

$$
\mathscr{I}_{\text {prod }}^{q l} \equiv \frac{\operatorname{Det}(\mathscr{U} \mathscr{D})}{\operatorname{Det}(\mathscr{N} \mathscr{L})} g_{1}^{-\frac{96}{41}} g_{3}^{-\frac{96}{7}} ; \quad \mathscr{I}_{\text {comm }}^{q l} \equiv \frac{\operatorname{Det}^{3}[\mathscr{U}, \mathscr{D}] \operatorname{Det}[\mathscr{N}, \mathscr{L}]}{\operatorname{Det}^{3}(\mathscr{U} \mathscr{D}) \operatorname{Det}^{\frac{5}{4}}(\mathscr{N} \mathscr{L})} g_{1}^{-\frac{81}{82}} g_{2}^{\frac{81}{38}}
$$

\section{References}

[1] D. A. Demir, Renormalization group invariants in the MSSM and its extensions, JHEP 11 (2005) 003, (arXiv:hep-ph/0408043v3).

[2] L.-x. Liu, Renormalization Invariants and Quark Flavor Mixings, Int. J. Mod. Phys. A25 (2010) 4975, (arXiv:0910.1326v2[hep-ph]).

[3] Z.-z. Xing and S. Luo, Radiative Corrections to the CKM Unitarity Triangles, J. Phys. G 37 (2010) 075018 (arXiv:0912.4593v2[hep-ph]).

[4] S Chang and T. K. Kuo, Renormalization invariants of the neutrino mass matrix, Phys. Rev. D 66, (2002) 111302 (arXiv:hep-ph/0205147v1).

[5] P. F. Harrison, R. Krishnan and W. G. Scott, Exact One-Loop Evolution Invariants in the Standard Model, Phys. Rev. D, 82 (2010) 096004 (arXiv:1007.3810 [hep-ph]).

[6] M. E. Machacek and M. T. Vaughn, Two-loop renormalization group equations in a general quantum field theory (II). Yukawa couplings, Nuclear Physics B, 236 (1984) 221.

[7] Z.-z. Xing, H. Zhang and S. Zhou, Updated Values of Running Quark and Lepton Masses Phys. Rev. D 77 (2008) 113016 (arXiv:0712.1419v3[hep-ph]). 